\title{
Correction to: Enabling Digital Transformation Through Cognitive Robotic Process Automation at Deutsche Telekom Services Europe
}

Christian Czarnecki, Chin-Gi Hong, Manfred Schmitz, and Christian Dietze

\section{Correction to:}

Chapter 7 in: N. Urbach et al. (eds.), Digitalization Cases Vol. 2, Management for Professionals, https://doi.org/10.1007/978-3-030-80003-1_7

Owing to an oversight, an incorrect affiliation was captured for Christian Czarnecki when published initially. This has now been corrected to reflect as "FH Aachen, Aachen, Germany”. 